\title{
VANISHING OF TOP EQUIVARIANT CHERN CLASSES OF REGULAR EMBEDDINGS*
}

\author{
MICHEL BRION ${ }^{\dagger}$ AND IVAN KAUSZ
}

Key words. Newstead-Ramanan conjecture, regular embedding, logarithmic tangent bundle, equivariant Chern class

AMS subject classifications. Primary 14H60, 14 M17; Secondary 55N25, 55R91

1. Introduction. Let $G$ be a connected affine algebraic group and let $X$ be a regular $G$-variety in the sense of [BDP] (recalled in Definition 2.2 below). The variety $X$ contains an open orbit $G / H$ whose complement $D$ is a strictly normal crossing divisor in $X$. In this note we show the following vanishing result for rational equivariant Chern classes of the bundle of logarithmic differentials on the variety $X$ :

$$
c_{i}^{G}\left(\Omega_{X}^{1}(\log D)\right)=0 \quad \text { for } i>\operatorname{dim}(X)-\operatorname{rk}(G)+\operatorname{rk}(H) .
$$

The motivation for this vanishing result originated in the second author's interest in a higher rank generalization of Gieseker's proof $[\mathrm{G}]$ of the Newstead-Ramanan conjecture. The conjecture (or rather its higher rank generalization) says that for coprime $r$ and $d$ and for $g \geq 1$ the Chern classes of the tangent bundle of the moduli space of stable vector bundles of rank $r$ and degree $d$ on a curve of genus $g$ vanish in degrees larger than $r(r-1)(g-1)$ (cf. [EK], bottom of page 844).

In order to explain the relationship between this conjecture and the result proven here let us first sketch Gieseker's degeneration of moduli spaces of vector bundles.

Let $B$ a smooth curve, which serves as the base scheme of the degeneration. Let $\mathcal{X} \rightarrow B$ be a proper flat family of algebraic curves of genus $g \geq 2$ which is smooth outside a point $x \in B$. Assume that the fiber $X$ over $x$ is irreducible with a unique singular point $p$ which is an ordinary double point. Let $\tilde{X}$ be the normalization of $X$. Let $r$ be a positive integer and let $d$ be an integer prime to $r$. Then there exists a variety $M(\mathcal{X} / B)$ proper and flat over $B$ such that

- the fiber of $M(\mathcal{X} / B) \rightarrow B$ over any point $y \in B \backslash\{x\}$ is the moduli space $M(Y)$ of stable vector bundles of rank $r$ and degree $d$ on the curve $Y=\mathcal{X}_{y}$,

- the variety $M(\mathcal{X} / B)$ is nonsingular and its fiber $M(X)$ over $x$ is a normal crossing divisor in $M(\mathcal{X} / B)$.

- Let $M(\tilde{X})$ be the moduli space of rank $r$ degree $d$ vector bundles on the curve $\tilde{X}$. Then there is a principal $\mathrm{GL}_{r} \times \mathrm{GL}_{r}$-bundle $P$ on $M(\tilde{X})$, and a smooth $\mathrm{GL}_{r} \times \mathrm{GL}_{r}$-equivariant compactification of $\mathrm{GL}_{r}=\left(\mathrm{GL}_{r} \times \mathrm{GL}_{r}\right) / \operatorname{diag}\left(\mathrm{GL}_{r}\right)$ which we denote by $\mathrm{KGL}_{r}$, such that the normalization $\tilde{M}$ of $M(X)$ is birationally equivalent to the locally trivial $\mathrm{KGL}_{r}$-fibration

$$
f: M^{\prime}:=P \times \mathrm{GL}_{r} \times \mathrm{GL}_{r} \mathrm{KGL}_{r} \rightarrow M(\tilde{X})
$$

associated to the principal bundle $P \rightarrow M(\tilde{X})$.

\footnotetext{
*Received August 25, 2005; accepted for publication October 13, 2005.

†Université de Grenoble I, Institut Fourier, BP 74, 38402 Saint-Martin d'Hères, France (Michel.Brion@ujf-grenoble.fr).

${ }^{\ddagger}$ NWF I - Mathematik, Universität Regensburg, 93040 Regensburg, Germany (ivan.kausz@ mathematik.uni-regensburg.de). The second author has been partially supported by the DFG.
} 
(cf. [NS], [Se], [K2], [KL]). Summarizing, we have a diagram of varieties as follows:

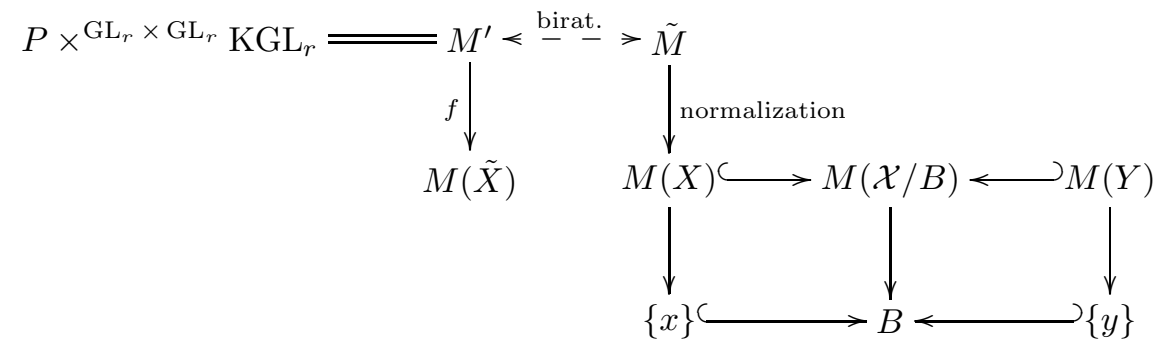

The conjecture of Newstead-Ramanan holds trivially for $g=1$, since the moduli space of vector bundles of rank $r$ and degree $d$ on an elliptic curve $E$ is isomorphic to $E$ itself (cf. [T]). Gieseker's idea was to use the above diagram (in the rank two case) to make induction on the genus $g$ (observe that the genus of $\tilde{X}$ is $g-1$ ).

Let $D^{\prime}$ be the complement of $P \times \mathrm{GL}_{r} \times \mathrm{GL}_{r} \mathrm{GL}_{r}$ in $M^{\prime}$ and let $\tilde{D}$ be the preimage of the singular locus of $M(X)$. Then $D^{\prime}$ and $\tilde{D}$ are normal crossing divisors in $M^{\prime}$ and $\tilde{M}$ respectively and they are proper transforms of each other by the birational correspondence $M^{\prime} \leftrightarrow \tilde{M}$.

The induction step consists in proving the following three implications:

Induction hypothesis

$\stackrel{(1)}{=}>$ Vanishing of Chern classes of $\Omega_{M^{\prime}}^{1}\left(\log D^{\prime}\right)$ in degrees larger than $r(r-1)(g-1)$

$\stackrel{(2)}{=}>$ Vanishing of Chern classes of $\Omega_{\tilde{M}}^{1}(\log \tilde{D})$ in degrees larger than $r(r-1)(g-1)$

$\stackrel{(3)}{=}>$ Vanishing of Chern classes of $\Omega_{Y}^{1}$ in degrees larger than $r(r-1)(g-1)$

The third implication is not difficult and follows from results already proven by Gieseker $[\mathrm{G}]$. The most difficult part is the second implication. It has been proven up to now only in the case $r=2$ by Gieseker [G]. In the case $r=3 \mathrm{Kiem}$ and Li [KL] were only able to prove the second implication with the slightly weaker bound $6 g-5$ instead of $6(g-1)$. In both papers $[\mathrm{G}]$ and $[\mathrm{KL}]$ the step $(2)$ is carried through by means of a detailed study of flips connecting the varieties $M^{\prime}$ and $\tilde{M}$.

It is the first implication, where the result of this note comes to a bearing. Namely, by Example 2.3 the $\mathrm{GL}_{r} \times \mathrm{GL}_{r}$-equivariant embedding $\mathrm{GL}_{r} \subset \mathrm{KGL}_{r}$ is regular, and applying Corollary 2.6 to this case it follows that $c_{i}\left(\Omega_{M^{\prime} / M(\tilde{X})}^{1}\left(\log D^{\prime}\right)\right)=0$ for $i>$ $r(r-1)$. On the other hand, the induction hypothesis implies $c_{i}\left(\Omega_{M(\tilde{X})}^{1}\right)=0$ for $i>r(r-1)(g-2)$. Thus the vanishing of the Chern classes of $\Omega_{M^{\prime}}^{1}\left(\log D^{\prime}\right)$ in degrees larger than $r(r-1)(g-1)$ follows from the exact sequence

$$
0 \rightarrow f^{*} \Omega_{M(\tilde{X})}^{1} \rightarrow \Omega_{M^{\prime}}^{1}\left(\log D^{\prime}\right) \rightarrow \Omega_{M^{\prime} / M(\tilde{X})}^{1}\left(\log D^{\prime}\right) \rightarrow 0 .
$$

It should be noted that in the papers $[\mathrm{G}]$ and $[\mathrm{KL}]$ the implication (1) is proved (in the rank two and three case) in a rather ad hoc way by using the fact that $f$ : $M^{\prime} \rightarrow M(\tilde{X})$ can be represented as a succession of blowing ups of a projective bundle. In principle this could be done also in the higher rank case, but as can be seen from the rank three case treated in $[\mathrm{KL}]$, the computations become very involved with increasing rank. 
Our Corollary 2.7 establishes the vanishing in the given range of the usual (nonequivariant) Chern classes of the bundle of logarithmic differential forms on a regular group embedding $G \hookrightarrow X$. This statement is proven by different methods in a recent paper of Valentina Kiritchenko (cf. [Ki] Lemma 3.6 and Proposition 4.4) where also the non-equivariant case of our Theorem 2.4 is mentioned (cf. [Ki] $\S 5$ ).

\section{Definitions and statement of the Theorem.}

Definition 2.1. Let $G$ be a topological group and let $X$ be a topological $G$-space. Let $F \rightarrow X$ be a $G$-linearized complex topological vector bundle. Let $E_{G} \rightarrow B_{G}$ be the universal $G$-bundle over the classifying space of $G$. The $G$-equivariant rational Chern class

$$
c_{i}^{G}(F) \in H_{G}^{2 i}(X):=H^{2 i}\left(E_{G} \times{ }^{G} X, \mathbb{Q}\right)
$$

of the bundle $F$ is by definition the Chern class of the vector bundle

$$
E_{G} \times{ }^{G} F \rightarrow E_{G} \times{ }^{G} X,
$$

where $E_{G} \times{ }^{G} F$ and $E_{G} \times{ }^{G} X$ are the quotients of $E_{G} \times F$ and $E_{G} \times X$ respectively by the diagonal action of $G$.

By construction, the pull-back of $c_{i}^{G}(F)$ to $X$ (regarded as a fiber of the map $\left.E_{G} \times{ }^{G} X \rightarrow B_{G}\right)$ is the usual Chern class $c_{i}(F)$.

Definition 2.2. Let $G$ be a connected affine algebraic group and let $X$ be an algebraic variety on which $G$ acts with an open dense orbit $\Omega$. The $G$-variety $X$ is called regular, if it satisfies the following conditions (see [BDP]):

1. The closure of every $G$-orbit is smooth.

2. Any orbit closure $Y \neq X$ is the transversal intersection of the orbit closures of codimension one containing $Y$.

3. The isotropy group of any point $x \in X$ has a dense orbit in the normal space to the orbit $G \cdot x$ in $X$.

EXAMPLE 2.3. Let $r \geq 1$ and let $G=\mathrm{GL}_{r} \times \mathrm{GL}_{r}$. We claim that the compactification $X:=\mathrm{KGL}_{r}$ of $\mathrm{GL}_{r}$ defined in [K1] is regular in the above sense if considered as a $G$-variety. Indeed, properties (1) and (2) follow directly from loc. cit. $\S 9$.

It remains to show property (3). For this let $S \subseteq \mathrm{GL}_{r}$ be the maximal torus of diagonal matrices and let $S \subset \bar{S}_{0}$ be the smooth torus embedding which in loc. cit. $\S 4$ we denoted by $T \subset \tilde{T}$ and which is an $S$-invariant open subset of the closure $\bar{S}$ in $X$. From the description of the orbits in loc. cit. $\S 9$ and the results in loc. cit. $\S 4$ it follows immediately that the intersection with $\bar{S}_{0}$ of any $G$-orbit of a certain codimension in $X$ is a unique $S$-orbit of the same codimension in $\bar{S}_{0}$ (or $\bar{S}$ ).

Therefore, to check property (3) it suffices to consider points $x \in \bar{S}_{0}$. Furthermore, it follows that the canonical map

$$
\left(N_{S \cdot x / \bar{S}_{0}}\right)_{x} \rightarrow\left(N_{G \cdot x / X}\right)_{x}
$$

of normal spaces at $x$ is an $S_{x}$-equivariant isomorphism. Being a smooth torus embedding, the $S$-variety $\bar{S}_{0}$ is regular in the above sense. Indeed, to see this one is immediately reduced to the case $\mathbb{G}_{m}^{n} \hookrightarrow \mathbb{A}^{n}$ where the assertion is clear. In particular, the isotropy subgroup $S_{x}$ acts with a dense orbit on $\left(N_{S \cdot x / \bar{S}_{0}}\right)_{x}$. Thus also $G_{x}$ acts with a dense orbit on $\left(N_{G \cdot x / X}\right)_{x}$. 
Let $X$ be a regular $G$-variety. Then the complement $D \subset X$ of the open orbit in $X$ is a strict normal crossing divisor. Recall that the sheaf of logarithmic differential forms $\Omega_{X}^{1}(\log D)$ is the locally free subsheaf of $\Omega_{X}^{1} \otimes_{\mathcal{O}_{X}} \mathbb{C}(X)$ generated in a neighbourhood of a point $x \in X$ by the differentials

$$
\frac{d f_{1}}{f_{1}}, \ldots, \frac{d f_{m}}{f_{m}}, d f_{m+1}, \ldots, d f_{n},
$$

where $f_{1}, \ldots, f_{n}$ are local coordinates at the point $x$ such that the divisor $D$ is given by the equation $f_{1} f_{2} \ldots f_{m}=0$. As can be easily seen, this definition is independent of the choice of the coordinate system.

The dual of $\Omega_{X}^{1}(\log D)$ is the subsheaf $T_{X}(-\log D)$ of the tangent sheaf $T_{X}$ locally at $x$ generated by the vector fields

$$
f_{1} \frac{\partial}{\partial f_{1}}, \ldots, f_{m} \frac{\partial}{\partial f_{m}}, \frac{\partial}{\partial f_{m+1}}, \ldots, \frac{\partial}{\partial f_{n}} .
$$

In more geometric terms, it is the subsheaf of the tangent sheaf whose sections consist of vector fields which are tangent to all the components of $D$. Since the components of $D$ are $G$-invariant, it is clear from this description that $T_{X}(-\log D)$ inherits a natural $G$-linearization from the one on $T_{X}$. Thus $\Omega_{X}^{1}(\log D)$ is $G$-linearized as well.

The main result of this note is the following:

THEOREM 2.4. Let $G$ be a connected affine algebraic group over the field of complex numbers and let $X$ be a complex regular $G$-variety. Let $H \subseteq G$ be the isotropy group of a point in the open orbit $\Omega \subseteq X$ and let $D:=X \backslash \Omega$ be the boundary divisor. Then the $G$-equivariant rational Chern classes of the bundle $\Omega_{X}^{1}(\log D)$ of logarithmic differentials vanish in degrees larger than

$$
\operatorname{dim}(X)-\operatorname{rk}(G)+\operatorname{rk}(H) .
$$

The proof will be given in the next section. We note that the corresponding statement over an algebraically closed field of arbitrary characteristic holds true as well with the same proof if one replaces rational equivariant cohomology with the equivariant Chow ring with rational coefficients.

The following is an immediate consequence:

Corollary 2.5. Let $G$ be a complex connected affine algebraic group and let $G=(G \times G) / \operatorname{diag}(G) \hookrightarrow X$ be a regular $G \times G$-equivariant embedding with boundary divisor $D$. Then we have $c_{i}^{G \times G}\left(\Omega_{X}^{1}(\log D)\right)=0$ for $i>\operatorname{dim}(G)-\operatorname{rk}(G)$.

For the application mentioned in the introduction, we state the following

Corollary 2.6. Let $G, X$ and $D$ be as in 2.5. Let $M$ be a complex variety and let $P$ be a principal $G \times G$-bundle over $M$. Let

$$
\mathcal{X}:=P \times^{G \times G} X \quad \text { and } \quad \mathcal{D}:=P \times{ }^{G \times G} D \subset \mathcal{X}
$$

be the associated locally trivial $X$-fibration and $D$-fibration over $M$ respectively. Then the Chern classes of $\Omega_{\mathcal{X} / M}^{1}(\log \mathcal{D})$ vanish in degrees larger than $\operatorname{dim}(G)-\operatorname{rk}(G)$. 
Proof. Let $M \rightarrow B_{G \times G}$ be a classifying map for the bundle $P$. Then we have cartesian diagrams
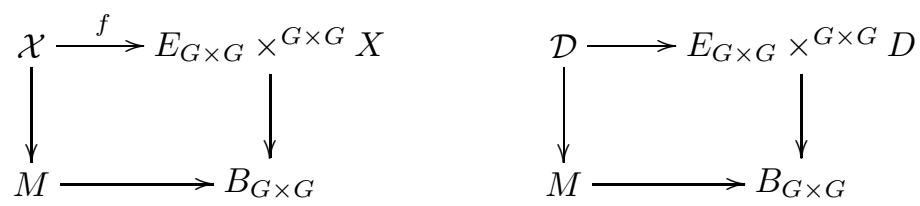

and $\Omega_{\mathcal{X} / M}^{1}(\log \mathcal{D})$ is the pull back via $f$ of the vector bundle

$$
E_{G \times G} \times{ }^{G \times G} \Omega_{X}^{1}(\log D) .
$$

Therefore we have

$$
c_{i}\left(\Omega_{\mathcal{X} / M}^{1}(\log \mathcal{D})\right)=f^{*} c_{i}\left(E_{G \times G} \times{ }^{G \times G} \Omega_{X}^{1}(\log D)\right)=f^{*} c_{i}^{G \times G}\left(\Omega_{X}^{1}(\log D)\right)
$$

and the result follows from Corollary 2.5.

Corollary 2.7. With the notation of Corollary 2.5, the usual Chern classes of $\Omega_{X}^{1}(\log D)$ vanish in degrees $>\operatorname{dim}(G)-\operatorname{rk}(G)$.

Proof. This is a special case of Corollary 2.6: Take $M$ to be a point.

3. Proof of the theorem. The first step in the proof of the theorem is the case of empty boundary divisor:

Proposition 3.1. Theorem 2.4 holds in the case when $X=G / H$.

Proof. We have to show the vanishing of the $G$-equivariant Chern classes of the cotangent bundle $\Omega_{X}^{1}$ in the given range. Let $x \in X$ be the point represented by the unit element in $G$. The fiber $V$ of $\Omega_{X}^{1}$ at $x$ is an $H$-module. In fact we have

$$
V=(\operatorname{Lie}(G) / \operatorname{Lie}(H))^{\vee},
$$

where the action of $H$ on the right hand side is induced by the adjoint action of $H$ on $\operatorname{Lie}(G)$.

Since $H$ operates freely on the contractible space $E_{G}$, we may take $E_{H}=E_{G}$ and $B_{H}=E_{G} / H=E_{G} \times{ }^{G} X$. Since $\Omega_{X}^{1}=G \times{ }^{H} V$, it follows that the vector bundle $E_{G} \times{ }^{G} \Omega_{X}^{1} \rightarrow E_{G} \times{ }^{G} X$ can be identified with the vector bundle

$$
\mathcal{V}:=E_{H} \times{ }^{H} V \rightarrow B_{H} .
$$

Thus we have to show the vanishing of the Chern classes of $\mathcal{V}$ in degrees larger than

$$
\operatorname{dim}(X)-\operatorname{rk}(G)+\operatorname{rk}(H) .
$$

Let $S_{H}$ be a maximal torus of $H$ and let $X^{*}\left(S_{H}\right)$ be its character group. By the results of [H] Chapter III, $\S 1$, the map $B_{S_{H}} \rightarrow B_{H}$ induces an injection

$$
H^{*}\left(B_{H}, \mathbb{Q}\right) \hookrightarrow H^{*}\left(B_{S_{H}}, \mathbb{Q}\right) \stackrel{\sim}{\rightarrow} \operatorname{Sym}_{\mathbb{Q}}\left(X^{*}\left(S_{H}\right) \otimes \mathbb{Q}\right) .
$$

Now if we denote by $\alpha_{1}, \ldots, \alpha_{\operatorname{dim} X} \in X^{*}\left(S_{H}\right)$ the weights of $V$ considered as an $S_{H}$-module, then the total Chern class of $\mathcal{V}$ is mapped to the product

$$
\prod_{i=1}^{\operatorname{dim} X}\left(1+\alpha_{i}\right) \in \operatorname{Sym}_{\mathbb{Q}}\left(X^{*}\left(S_{H}\right) \otimes \mathbb{Q}\right) \text {. }
$$


But $S_{H}$ acts trivially on the subspace

$$
\operatorname{Lie}\left(S_{G}\right) / \operatorname{Lie}\left(S_{H}\right) \subseteq \operatorname{Lie}(G) / \operatorname{Lie}(H)=V^{\vee},
$$

where $S_{G}$ denotes a maximal torus of $G$ containing $S_{H}$. Therefore at least as many as $\operatorname{dim}\left(\operatorname{Lie}\left(S_{G}\right) / \operatorname{Lie}\left(S_{H}\right)\right)$ of the $\alpha_{i}$ vanish and the total Chern class of $\mathcal{V}$ is of degree at most

$$
\operatorname{dim}(X)-\operatorname{dim}\left(\operatorname{Lie}\left(S_{G}\right) / \operatorname{Lie}\left(S_{H}\right)\right)=\operatorname{dim}(X)-\operatorname{rk}(G)+\operatorname{rk}(H)
$$

Proposition 3.2. Let $G$ be a connected affine algebraic group and let $X$ be a regular $G$-variety. Let $x, y \in X$ be two points and let $G \cdot x$ and $G \cdot y$ be the corresponding $G$-orbits in $X$. Assume that the orbit closure $\overline{G \cdot y}$ is contained in $\overline{G \cdot x}$ as a divisor. Let $G_{x}$ and $G_{y}$ be the isotropy subgroups of the points $x$ and $y$. Then

$$
\operatorname{rk}\left(G_{x}\right) \geq \operatorname{rk}\left(G_{y}\right)-1
$$

Proof. Since $\overline{G \cdot x}$ is again a regular $G$-variety, we may assume without loss of generality that $\overline{G \cdot x}=X$. This simplifies notation a bit.

Let $S_{y}$ be a maximal subtorus of $G_{y}$. It acts on the tangent space $T_{y}(X)$ and leaves the codimension-one subspace $T_{y}(G \cdot y)$ invariant. Therefore there is a onedimensional $S_{y}$-invariant subspace $\ell$ in $T_{y}(X)$ such that

$$
T_{y}(X)=T_{y}(G \cdot y) \oplus \ell .
$$

Let $\chi: S_{y} \rightarrow \mathbb{C}^{\times}$be the character associated to the one-dimensional $S_{y}$-module $\ell$ and let $S$ be the connected component of the identity in $\operatorname{ker}(\chi)$. We claim that there is a point $x_{0} \in G \cdot x$ which is fixed by $S$.

Indeed, since $X$ is normal, by a result of Sumihiro ([Su]) there is an affine open neighbourhood $U \cong \operatorname{Spec}(R)$ of $y$ in $X$ which is invariant under the action of $S$. Let $\mathfrak{m} \subset R$ be the maximal ideal corresponding to the point $y \in U$. Since $S$ is a torus, there is an $S$-equivariant section

$$
\varphi: \mathfrak{m} / \mathfrak{m}^{2} \rightarrow \mathfrak{m}
$$

of the surjection $\mathfrak{m} \rightarrow \mathfrak{m} / \mathfrak{m}^{2}$. The map $\varphi$ induces an $S$-equivariant map $\operatorname{Sym}\left(\mathfrak{m} / \mathfrak{m}^{2}\right) \rightarrow R$ and thus an $S$-equivariant morphism

$$
f: U=\operatorname{Spec}(R) \rightarrow \operatorname{Spec}\left(\operatorname{Sym}\left(\mathfrak{m} / \mathfrak{m}^{2}\right)\right)=T_{y}(X) .
$$

By construction, $f$ induces an isomorphism from the tangent space of $U$ at $y$ to the tangent space of $T_{y}(X)$ at 0 . Therefore after possibly restricting $f$ to a smaller $S$-invariant neighbourhood of $y$, we may assume that $f$ is étale.

Let $Z$ be the preimage by $f$ of the line $\ell$. Since $\ell$ is transversal to the tangent directions of $G \cdot y$, it follows that $Z$ is not contained in $G \cdot y$. Let $x_{0} \in Z \backslash G \cdot y$.

Let us collect what we know about the point $x_{0}$. The image $f\left(x_{0}\right)$ of $x_{0}$ in $T_{y}(X)$ is contained in $\ell$ and is thus fixed by $S$. Since $f$ is $S$-equivariant, the group $S$ acts on the fiber of $f$ at $f\left(x_{0}\right)$. But this fiber contains only finitely many points and $S$ is connected, so $S$ must act trivially on the fiber. In particular, $x_{0}$ is a fixed point of $S$ contained in $G \cdot x$. This proves our claim. 
It follows that $S \subseteq G_{x_{0}}$ and consequently we have

$$
\operatorname{rk}\left(G_{x}\right)=\operatorname{rk}\left(G_{x_{0}}\right) \geq \operatorname{dim}(S) \geq \operatorname{dim}\left(S_{y}\right)-1=\operatorname{rk}\left(G_{y}\right)-1 .
$$

Corollary 3.3. Let $G$ be a connected affine algebraic group and let $X$ be a regular $G$-variety. Let $H \subset G$ be the isotropy group of a point in the open orbit. Then for any point $y \in X$ with isotropy group $G_{y}$ we have the inequality

$$
\operatorname{dim}(H)-\operatorname{rk}(H) \leq \operatorname{dim}\left(G_{y}\right)-\operatorname{rk}\left(G_{y}\right)
$$

Proof. Let $c$ be the codimension in $X$ of the orbit $G \cdot y$. There is a sequence of points $x_{0}, x_{1}, \ldots, x_{c}$ in $X$ such that $x_{0}$ is contained in the open orbit and $x_{c}=y$, and such that for $i=1, \ldots, c$ the orbit closure $\overline{G \cdot x_{i}}$ is a divisor in $\overline{G \cdot x_{i-1}}$. Applying Proposition $3.2 c$ times, it follows that

$$
\operatorname{rk}(H) \geq \operatorname{rk}\left(G_{y}\right)-c .
$$

On the other hand we have

$$
\operatorname{dim}(G)-\operatorname{dim}(H)-c=\operatorname{dim}(X)-c=\operatorname{dim}(G \cdot y)=\operatorname{dim}(G)-\operatorname{dim}\left(G_{y}\right)
$$

and therefore $\operatorname{dim}(H)=\operatorname{dim}\left(G_{y}\right)-c$. From this the inequality of Corollary 3.3 is immediate.

Proposition 3.4. Let $G$ be a connected affine algebraic group and let $X$ be a regular $G$-variety. Let $D \subset X$ be the boundary divisor. Let $Y=G \cdot y$ be a $G$-orbit of codimension $c$ in $X$. Then there is an exact sequence of $G$-linearized bundles on $Y$ as follows:

$$
\left.0 \rightarrow \Omega_{Y}^{1} \rightarrow \Omega_{X}^{1}(\log D)\right|_{Y} \rightarrow \mathcal{O}_{Y}^{\oplus c} \rightarrow 0
$$

Proof. This follows from [BB], 2.4.2. $\mathrm{B}$

Now we can prove Theorem 2.4. For each $G$-orbit $Y \subseteq X$ we have the restriction map $H_{G}^{*}(X) \rightarrow H_{G}^{*}(Y)$. Thus we get a homomorphism

$$
H_{G}^{*}(X) \rightarrow \prod_{Y} H_{G}^{*}(Y)
$$

where $Y$ runs through all $G$-orbits of $X$. By [BDP] Theorem 7 this map is injective.

Therefore it suffices to show that for any

$$
i>\operatorname{dim}(G)-\operatorname{dim}(H)-\operatorname{rk}(G)+\operatorname{rk}(H)
$$

and any $G$-orbit $Y=G \cdot y$ in $X$ the $i$-th $G$-equivariant rational Chern class of $\left.\Omega_{X}^{1}(\log D)\right|_{Y}$ vanishes. By Proposition 3.4 this is equivalent to the vanishing of the $i$-th $G$-equivariant rational Chern class of $\Omega_{Y}^{1}$.

Let $G_{y}$ be the isotropy group of the point $y$. From Corollary 3.3 we know that

$$
i>\operatorname{dim}(G)-\operatorname{dim}(H)-\operatorname{rk}(G)+\operatorname{rk}(H) \geq \operatorname{dim}(G)-\operatorname{dim}\left(G_{y}\right)-\operatorname{rk}(G)+\operatorname{rk}\left(G_{y}\right) .
$$

Proposition 3.1 yields now the vanishing of $c_{i}^{G}\left(\Omega_{Y}^{1}\right)$ as required. 
4. Added in Proof. After this paper has been written, Teleman and Woodward [TW] have published a proof of the Newstead-Ramanan conjecture by a different approach than the one we outlined in the introduction.

\section{REFERENCES}

[BB] F. BIEn AND M. BRIOn, Automorphisms and local rigidity of regular varieties, Compositio Mathematica, 104 (1996), pp. 1-26.

[BDP] E. Bifet, C. De Concini, C.Procesi, Cohomology of Regular Embeddings, Advances in Mathematics, 82 (1990), pp. 1-34.

[EK] R. EARL, F. KIRWAN, The Pontryagin rings of moduli spaces of arbitrary rank holomorphic bundles over a Riemann surface, J. London Math. Soc. (2), 60:3 (1999), pp. 835-846.

[G] D. GieseKer, A degeneration of the moduli space of stable bundles, J. Differential Geometry, 19 (1984), pp. 173-206.

[H] W. Y. Hsiang, Cohomological Theory of Topological Transformation Groups, Ergeb. der Math. 85, Springer-Verlag, New York, 1975.

[K1] I. KaUsz, A modular compactification of the general linear group, Documenta Mathematica, 5 (2000), pp. 553-594.

[K2] I. KAUsz, A Gieseker type degeneration of moduli stacks of vector bundles on curves, Transactions of the AMS, to appear.

[KL] Y.-H. KIEM AND J. LI, Vanishing of the top Chern classes of the moduli of vector bundles, preprint math.AG/0403033.

[Ki] V. Kiritchenko, Chern classes of compactifications of reductive groups, preprint math.AG/0411331.

[NS] D.S. Nagaraj And C.S. Seshadri, Degenerations of the moduli spaces of vector bundles on curves II, Proc. Indian Acad. Sci. Math. Sci., 109:2 (1999), pp. 165-201.

[Se] C.S. Seshadri, Degenerations of the moduli spaces of vector bundles on curves, School on Algebraic Geometry (Trieste, 1999), pp. 205-265, ICTP Lect. Notes, 1, Abdus Salam Int. Cent. Theoret. Phys., Trieste, 2000.

[Su] H. Suminiro, Equivariant completion J. Math. Kyoto Univ., 14 (1974), pp. 1-28.

[T] L.W. Tu, Semistable Bundles over an Elliptic Curve, Advances in Mathematics, 98 (1993), pp. $1-26$.

[TW] C. Teleman and C.T. Woodward, The Newstead-Ramanan conjecture for Chern classes, preprint math.AG/0512486. 